\title{
Kerr beam self-cleaning in the telecom band
}

\author{
Yann Leventoux ${ }^{1}$, Geoffroy Granger ${ }^{1}$, Alexandre Parriaux ${ }^{2}$, Mathieu Jossent ${ }^{3}$, Laure Lavoute ${ }^{3}$, Dmitry \\ Gaponov $^{3}$, Alessandro Tonello ${ }^{1}$, Katarzyna Krupa ${ }^{2}$, Marc Fabert ${ }^{1}$, Guy Millot ${ }^{2}$, \\ Sébastien Février ${ }^{1}$, Stefano Wabnitz ${ }^{4}$, Vincent Couderc ${ }^{1}$ \\ 1. Université de Limoges, XLIM, UMR CNRS 7252, 123 av. A. Thomas, 87060 Limoges, France \\ 2. Université de Bourgogne Franche-Comté, ICB, UMR CNRS 6303, 9 av. A.Savary, 21078 Dijon, France \\ 3. Novae, ZI du Moulin Cheyroux, 87700 Aixe-sur-Vienne, France \\ 4. DIET, Sapienza Università di Roma, via Eudossiana 18, 00184 Rome, Italy
}

Multimode graded index (GRIN) fibers received a renewed interest in recent years, in particular for the development of new laser sources [1]. In many cases, the use of GRIN fibers is limited by multimodal propagation, leading to a spatially modulated intensity distribution (speckles) at the fiber output. Recent studies have found that quasi-single mode propagation can be recovered in GRIN fibers by the so-called Kerr self-cleaning effect [2]. It consists in the spontaneous recovery of the spatial beam quality, without any frequency shift [2] (as opposed to, e.g., Raman beam self-cleaning [3]). This nonlinear process was only observed so far at laser wavelengths around $1 \mu \mathrm{m}$, for peak power levels above a certain threshold value. In this communication, we show that Kerr beam selfcleaning also occurs in a GRIN fiber pumped with chirped pulses in the telecom band (1562 nm). At these wavelengths, the power threshold of the self-cleaning process is decreased by one order of magnitude with respect to pumping at $1 \mu \mathrm{m}$.

We have launched 200 ps pulses at $1562 \mathrm{~nm}$ with $10 \mathrm{~nm}$ bandwidth (at $-10 \mathrm{~dB}$ ) into a 50/125 GRIN fiber (50 $\mu \mathrm{m}$ core diameter, core-cladding index difference of 0.015 , numerical aperture of 0.2 ). The laser beam was coupled into a $12 \mathrm{~m}$ long piece of GRIN fiber by means of a short section of standard single mode fiber (SSMF). We observed the near-field intensity distribution at the output of the GRIN fiber for various input powers: in Fig. 1a we show the results against the launched peak power. As can be seen, the output intensity distribution shows a transition from a speckled pattern at low powers $(10 \mathrm{~W})$ into a bell-shaped pattern, resembling that of the fundamental mode of the GRIN fiber, as the input power is increased above a few hundred Watts. We have also calculated the correlation factor between the near-field intensity and that computed for the fundamental mode (Fig.1b). It is clear that the correlation factor increases with peak power up to nearly unity. Furthermore, we measured the spectrum at the output of the GRIN fiber for various peak powers. This shows that spatial energy transfer from the low-order modes to the fundamental mode appears well before the occurrence of any significant bandwidth broadening. This measurement confirms that the observed spatial beam reshaping is due to the Kerr self-cleaning process and cannot be attributed to Raman scattering effect or self-focusing.

It is worth noting that the observed beam reshaping appears in the telecom band with a peak power threshold which is more than ten times lower than the Kerr self-cleaning threshold, as observed in previous works carried out in the normal dispersion regime [3] (a few $\mathrm{kWs}$ ). We attribute this power threshold reduction to the relatively small number of modes excited at the input of the GRIN fiber when injecting the beam from the SSMF.
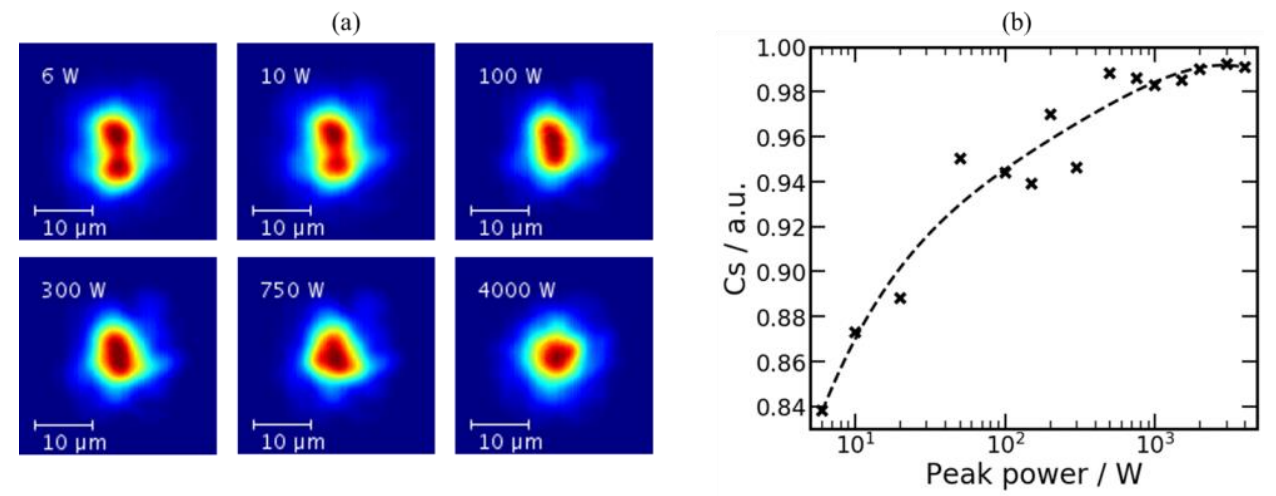

Fig. 1 (a) Near-field intensity distribution at the output of $12 \mathrm{~m}$ of GRIN fiber versus input peak powers. Scale bar: $10 \mu \mathrm{m}$. (b) Intensity correlation Cs (dots; dashed line: guide for the eye) between measured near-field intensity and calculated $\mathrm{LP}_{01}$ intensity as a function of input peak power.

\section{References}

[1] L.G. Wright, et al. "Spatiotemporal mode-locking in multimode fiber lasers." Science 358.6359 (2017): $94-97$.

[2] K. Krupa, et al. "Spatial beam self-cleaning in multimode fibres." Nature Photonics 11.4 (2017): 237.

[3] A.B. Grudinin, et al. "Nonlinear mode coupling in multimode optical fibers; excitation of femtosecond-range stimulated-Raman-scattering solitons." JETP Lett 47.6 (1988): 356. 